\title{
Pembrolizumab for treatment of advanced gastric and gastroesophageal junction adenocarcinoma
}

\author{
Smita S Joshi ${ }^{1}$, Steven B Maron ${ }^{1}$ \& Daniel V Catenacci*,2 \\ ${ }^{1}$ Section of Hematology/Oncology, University of Chicago, 5841 South Maryland Avenue, Chicago, IL 60637, USA \\ ${ }^{2}$ The University of Chicago Medical Center \& Biological Sciences, 900 East 57th Street, KCBD Building, Office 7128, Chicago, IL \\ 60637, USA \\ * Author for correspondence: dcatenac@medicine.bsd.uchicago.edu
}

Pembrolizumab is a monoclonal antibody directed against PD-1 that is US FDA approved for treatment of advanced PD-L1 positive gastric and gastroesophageal junction adenocarcinoma in patients who have progressed on at least two prior lines of chemotherapy. This article summarizes the clinical evidence regarding safety, tolerability and efficacy of pembrolizumab in this setting. In addition, this article describes the investigational use of pembrolizumab as first- and second-line therapy and in combination with other treatments. Finally, this review compares other checkpoint inhibitors to pembrolizumab for the treatment of this disease, and explores predictive biomarkers of response to PD-1 blockade.

First draft submitted: 30 August 2017; Accepted for publication: 13 October 2017; Published online: 2 November 2017

Keywords: esophageal cancer • esophagogastric cancer • gastric cancer • gastroesophageal cancer • immune checkpoint blockade • immunotherapy • MK-3475 • pembrolizumab • PD-1 • PD-L1

Gastric and esophageal cancer are the third and sixth most common causes of cancer mortality worldwide [1]. Taken together, gastroesophageal cancer is the second leading cause of cancer mortality. In the USA, 28,000 new cases of gastric cancer (GC) are estimated in 2017 with 10,960 deaths attributed to the disease [2]. Similarly, 16,940 new cases of esophageal cancer and 15,690 deaths are predicted [2]. While the incidence of both esophageal squamous cell carcinoma (SCC) and distal gastric adenocarcinoma (AC) is decreasing in this country, the rate of AC of the proximal esophagogastric junction (EGJ; including esophageal AC Siewert I, junction Siewert II and gastric cardia Siewert III) is increasing reflecting the rising prevalence of implicated risk factors including obesity and gastroesophageal reflux [3,4].

Patients with distal gastric AC and proximal EGJ AC, together known as gastroesophageal adenocarcinoma (GEA), most often present with inoperable or metastatic disease. Even when identified early and able to undergo curative intent resection, a majority (55-60\%) will recur over the next 5 years [5]. Therefore, we are most typically faced with the challenge of de novo or recurrent advanced disease. A number of first-line doublet and triplet palliative therapies are available, but fluoropyrimidine $(5 \mathrm{FU})$ and platinum doublet cytotoxic chemotherapy has emerged as the choice regimen for most treatment centers. Second- and third-line palliative cytotoxic therapies with taxaneor irinotecan-based regimens are routinely used. However, despite optimal chemotherapy, median overall survival (OS) for metastatic disease remains dismal at 11-12 months, highlighting the need for novel treatments [6,7]. Recent advances including the use of trastuzumab (a HER2 targeting monoclonal antibody) in the first-line setting for HER2 amplified tumors, as well as ramucirumab (a VEGFR-2 antagonist) for second-line therapy in unselected patients have expanded the armamentarium of active therapies. In the ToGA trial, the addition of trastuzumab to first-line 5FU plus platinum chemotherapy improved OS by 2.7 months in patients with HER2-positive advanced GEA [8]. In the REGARD and RAINBOW trials, the use of ramucirumab in the second-line setting improved OS by 1.4 months when used as monotherapy and by 2.2 months when used in combination with paclitaxel $[9,10]$. Unfortunately, studies investigating other targeted drugs have been disappointing. When added to chemotherapy, lapatinib (inhibitor of HER2 and EGFR) has been unsuccessful at prolonging survival in both the first- and second-

Future Medicine 
line settings in HER2-positive patients [11,12]. Trastuzumab emtansine (antibody-drug conjugate of trastuzumab linked to the tubulin inhibitor emtansine) has also failed to show a survival benefit in the second-line setting of HER2-positive patients [13]. Finally, the addition of pertuzumab (an HER2 targeting monoclonal antibody) to trastuzumab plus chemotherapy has not conferred a survival benefit in the first-line setting of HER2-positive patients [14]. Regimens using EGFR inhibitors have also failed to improve survival in the first- and subsequent-line settings in unselected patients [15-17]. The addition of rilotumumab or onartuzumab (monoclonal antibodies that interfere with MET/HGF signaling) to chemotherapy in the first-line setting has not been shown to improve outcomes in MET-positive patients [18,19]. There is a clear need to improve on outcomes of patients with advanced GEA.

In recent years, immune checkpoint blockade has emerged as an exciting therapeutic strategy in several malignancies. The development of monoclonal antibodies that inhibit PD-1, PD-L1 and CTLA-4 have shown dramatic responses and clinical benefit across multiple tumor types. Pembrolizumab is an antibody directed toward PD- 1 that is already US FDA approved for use in melanoma, non-small cell lung cancer (NSCLC), head and neck squamous cell cancer, classical Hodgkin lymphoma, urothelial carcinoma and any microsatellite instability-high (MSI-H) cancer including GEA [20]. In September 2017, pembrolizumab was approved by the FDA for the third-line (or higher) treatment of advanced PD-L1 positive GEA [21]. Recently published data have demonstrated a manageable safety profile and promising antitumor effect of pembrolizumab in GEA. Additionally, early evidence suggests that tumors positive for Epstein-Barr virus (EBV) may be particularly sensitive to immune checkpoint inhibition [22]. This review will examine the pharmacology, safety/tolerability and efficacy of pembrolizumab in treatment of GEA, with particular focus on predictive biomarkers.

\section{Pharmacology}

\section{Chemistry}

Pembrolizumab, formerly known as MK-3475 and lambrolizumab, is a humanized monoclonal IgG4 kappa antibody with an approximate weight of $149 \mathrm{kDa}$ that prevents PD-1 binding with PD-L1 and PD-L2 [20].

\section{Mechanism of action}

The transmembrane receptor PD-1 is expressed on a variety of immune cells including T cells and is a checkpoint that modulates the immune response. The binding of PD-1 to its ligands PD-L1 and PD-L2 results in transduction of inhibitory signals that downregulate T-cell function, reducing T-cell ability to eliminate neoplastic cells [23]. PD-L1 is a transmembrane protein expressed on the surface of several tissue types including neoplastic cells. By expressing PD-L1 and co-opting the inhibitory signaling pathway, tumors can evade T-cell immune surveillance and destruction. Pembrolizumab is a monoclonal antibody that binds to PD-1 and blocks its binding with PD-L1 and PD-L2, thereby removing the physiologic 'brake' on an activated immune system and restoring antitumor response [20].

\section{Pharmacokinetics \& pharmacodynamics}

The first-in-human Phase I study of pembrolizumab showed that pembrolizumab was safe at doses ranging from $1-10 \mathrm{mg} / \mathrm{kg}$ every 2 weeks and that a dose of at least $2 \mathrm{mg} / \mathrm{kg}$ every 3 weeks was needed for robust clinical activity [24]. There was a linear serum exposure to pembrolizumab at $0.1-10 \mathrm{mg} / \mathrm{kg}$ steady state dosing [24]. Population pharmacokinetic modeling using pooled data from three KEYNOTE studies of 2195 patients with advanced melanoma, NSCLC and other solid tumors found that pembrolizumab has a low clearance $(0.22$ $\mathrm{L} /$ day) and small volume of distribution (central volume $3.48 \mathrm{~L}$, peripheral volume $4.06 \mathrm{~L}$ ) [25]. Bodyweight, age, sex, tumor type, tumor burden, renal impairment and hepatic impairment had no clinically relevant impact on pembrolizumab exposure [25]. Given the lack of influence of bodyweight, a fixed-dose regimen of $200 \mathrm{mg}$ every 3 weeks was adopted as this maintained exposures comparable to those of $2 \mathrm{mg} / \mathrm{kg}$ every 3 weeks (the initial approved dose of pembrolizumab) [26]. Based on pembrolizumab prescribing data from Merck (NJ, USA), the following variables have no clinically meaningful effect on pembrolizumab clearance: age (range: 15-94 years), sex, race $\left(94 \%\right.$ white), renal impairment (eGFR $\geq 15 \mathrm{ml} / \mathrm{min} / 1.73 \mathrm{~m}^{2}$ ), mild hepatic impairment (total bilirubin $\leq$ upper limit normal [ULN] and AST > ULN or total bilirubin 1- to 1.5-times ULN and any AST) and tumor burden [20]. Additional data are needed regarding effects of moderate and severe hepatic impairment on clearance. 
Table 1. Summary of reported Phase I and II studies of pembrolizumab in advanced gastric and gastroesophageal cancer.

\begin{tabular}{|c|c|c|c|c|}
\hline Study (year) & Study design & Efficacy findings & Safety findings & Ref. \\
\hline $\begin{array}{l}\text { Muro et al. (2016) } \\
\text { KEYNOTE-012 }\end{array}$ & $\begin{array}{l}\text { Phase } \mathrm{lb}, \mathrm{PEM} 10 \mathrm{mg} / \mathrm{kg} \mathrm{q} 2 \mathrm{w} \\
\text { Multicenter, international } \\
\mathrm{n}=39 \text { (GEA, PD-L1+) } \\
\text { All lines of therapy }\end{array}$ & $\begin{array}{l}\text { Response rate ( } 36 \text { evaluable): } \\
\text { - CR } 0 \% \\
\text { - PR } 22 \% \\
\text { - SD } 14 \% \\
\text { - PD } 53 \% \\
\text { - Median DoR } 40 \text { weeks } \\
\text { Survival: } \\
\text { - Median PFS } 1.9 \text { months } \\
\text { (95\% Cl: } 1.8-3.5 \text { ) } \\
\text { - Median OS } 11.4 \text { months } \\
\text { (95\% Cl: } 5.7-\text {-not reached) }\end{array}$ & $\begin{array}{l}\text { - Frequent any grade TRAE: fatigue }(18 \%) \text {, } \\
\text { decreased appetite }(13 \%) \text {, pruritus }(13 \%) \text {, } \\
\text { hypothyroidism }(13 \%) \text {, arthralgias }(10 \%) \\
\text { - Grade 3: fatigue }(5.1 \%) \text {, pemphigoid }(2.6 \%) \text {, } \\
\text { hypothyroidism }(2.6 \%) \text {, peripheral sensory } \\
\text { neuropathy }(2.6 \%) \\
\text { - Grade 4: pneumonitis }(2.6 \%) \\
\text { - No patients discontinued treatment due to TRAE }\end{array}$ & [31] \\
\hline $\begin{array}{l}\text { Doi et al. (2016) } \\
\text { KEYNOTE-028 } \\
\text { Esophageal carcinoma } \\
\text { cohort }\end{array}$ & $\begin{array}{l}\text { Phase Ib, PEM } 10 \mathrm{mg} / \mathrm{kg} \mathrm{q} 2 \mathrm{w} \\
\text { Multicenter, international } \\
\mathrm{n}=23 \text { (E SCC or EGJ AC, PD-L1+) } \\
2 \mathrm{~L} \text { or subsequent therapy }\end{array}$ & $\begin{array}{l}\text { Response rate: } \\
\text { - ORR (CR + PR) } 30 \% \\
\text { - SD } 13 \% \\
\text { - Median DoR } 40 \text { weeks } \\
\text { Survival: } \\
\text { - } 6 \text { months PFS: } 30 \% \\
-12 \text { months PFS: } 22 \%\end{array}$ & $\begin{array}{l}\text { - Any grade TRAEs: } 39 \% \text {, most commonly } \\
\text { decreased appetite }(13 \%) \\
\text { - Immune-mediated AEs: grade } 2 \text { hypothyroidism } \\
\text { (9\%), grade } 2 \text { adrenal insufficiency }(4 \%) \\
\text { - Grade } 3 \text { TRAEs: } 17 \%\end{array}$ & [32] \\
\hline $\begin{array}{l}\text { Fuchs et al. } \\
\text { Wainberg et al. (2017) } \\
\text { KEYNOTE-059 Cohort } 1\end{array}$ & $\begin{array}{l}\text { Phase II, PEM } 200 \text { mg q3w } \\
\text { Multicenter, international } \\
n=259 \text { (GEA) } \\
3 \text { L or subsequent therapy }\end{array}$ & $\begin{array}{l}\text { Response rate: } \\
\text { - ORR 12\% (PD-L1+ 16\%, PD-L1- } \\
6 \% \text { ) } \\
\text { Survival: } \\
\text { - Median PFS: } 2 \text { months } \\
\text { - Median OS: } 6 \text { months }\end{array}$ & $\begin{array}{l}\text { - TRAE } \geq \text { grade } 3: 18 \% \text {, resulting in } \\
\text { discontinuation in seven patients }(3 \%) \text { and death } \\
\text { in two patients }(1 \%)\end{array}$ & {$[34,35]$} \\
\hline $\begin{array}{l}\text { Bang et al. } \\
\text { Wainberg et al. (2017) } \\
\text { KEYNOTE-059 Cohort } 2\end{array}$ & $\begin{array}{l}\text { Phase II, PEM } 200 \mathrm{mg} \\
\mathrm{q} 3 \mathrm{w}+\mathrm{CIS}+5 \mathrm{FU} / \text { Capecitabine } \\
\text { Multicenter, international } \\
\mathrm{n}=25 \text { (GEA, HER2-) } \\
1 \mathrm{~L} \text { therapy }\end{array}$ & $\begin{array}{l}\text { Response rate: } \\
\text { - ORR } 60 \% \text { (PD-L1+ 73\%, PD-L1- } \\
38 \% \text { ) } \\
\text { Survival: } \\
\text { - Median PFS } 7 \text { months ( } 95 \% \\
\text { Cl: } 6-11 \text { ) } \\
\text { - Median OS } 14 \text { months ( } 95 \% \\
\text { Cl: } 9 \text {-not estimable) }\end{array}$ & $\begin{array}{l}\text { - TRAE } \geq \text { grade } 3: 76 \% \\
\text { - TRAEs led to discontinuation in three patients } \\
\text { - No fatal TRAEs }\end{array}$ & {$[35,37]$} \\
\hline $\begin{array}{l}\text { Catenacci et al. } \\
\text { Wainberg et al. (2017) } \\
\text { KEYNOTE-059 Cohort } 3\end{array}$ & $\begin{array}{l}\text { Phase II, PEM } 200 \text { mg q3w } \\
\text { Multicenter, international } \\
\mathrm{n}=31 \text { (GEA, PD-L1+, HER2-) } \\
1 \mathrm{~L} \text { therapy }\end{array}$ & $\begin{array}{l}\text { Response rate: } \\
\text { - CR } 3.2 \% \\
\text { - PR } 23 \% \\
\text { - Median DoR not reached } \\
\text { Survival: } \\
\text { - Median PFS } 3.3 \text { months }(95 \% \\
\text { Cl: } 2.0-6.0 \text { ) } \\
\text { - } 6 \text { months OS: } 73 \% \\
\text { - } 12 \text { months OS: } 62 \% \\
\text { - Median OS not reached }\end{array}$ & $\begin{array}{l}\text { - Any grade TRAEs: } 77 \% \\
\text { - TRAE } \geq \text { grade } 3: 23 \% \text {, of which one patient died } \\
\text { (pneumonitis) } \\
\text { - No patients discontinued treatment due to TRAE }\end{array}$ & {$[35,36]$} \\
\hline Chau et al. (2017) & $\begin{array}{l}\text { Phase la/b, PEM } 200 \mathrm{mg} \\
\mathrm{q} 3 \mathrm{w}+\mathrm{RAM} \\
\text { Multicenter, international } \\
\mathrm{n}=40 \text { (GEA) } \\
2 \mathrm{~L} \text { or subsequent therapy }\end{array}$ & $\begin{array}{l}\text { Response rate: } \\
\text { - PR } 7.5 \% \\
\text { - Disease control rate } 45 \% \\
\text { Survival: } \\
\text { - Median PFS } 2.1 \text { months (RAM } \\
8 \mathrm{mg} / \mathrm{kg} \text { on days } 1 \text { and } 8 \text { ) } \\
\text { - Median PFS } 2.6 \text { months (RAM } \\
10 \mathrm{mg} / \mathrm{kg} \text { on day } 1 \text { ) }\end{array}$ & $\begin{array}{l}\text { - Any grade TRAEs: } 78 \% \text {, most commonly fatigue } \\
(30 \%) \text {, infusion related reaction }(13 \%) \text {, decreased } \\
\text { appetite }(13 \%) \text {, pruritus }(10 \%) \text {, maculopapular } \\
\text { rash }(10 \%) \text {, hypertension }(10 \%) \\
\text { - TRAE grade } 3-4: 10 \% \text {, most commonly colitis } \\
(7.5 \%) \text { and hypertension }(7.5 \%) \\
\text { - One death due to TRAE (pneumocystis, } \\
\text { pneumonia and pulmonary sepsis) }\end{array}$ & [38] \\
\hline \multicolumn{5}{|c|}{$\begin{array}{l}1 \text { L: First line; } 2 \text { L: Second line; } 3 \text { L: Third line; AC: Adenocarcinoma; AE: Adverse event; CIS: Cisplatin; CR: Complete response; DoR: Duration of response; E: Esophageal; G: Gastric; } \\
\text { GEA: Gastric and esophagogastric adenocarcinoma; EGJ: Esophagogastric junction; ORR: Objective response rate; OS: Overall survival; PEM: Pembrolizumab; PFS: Progression free } \\
\text { survival; PR: Partial response; q2w: Every } 2 \text { weeks; q3w: Every } 3 \text { weeks; RAM: Ramucirumab; SCC: Squamous cell carcinoma; SD: Stable disease; TRAE: Treatment related adverse } \\
\text { event }\end{array}$} \\
\hline
\end{tabular}

\section{Phase I \& II evidence of pembrolizumab in GEA}

\section{Pembrolizumab monotherapy}

The first trial of pembrolizumab was performed in patients with advanced solid tumors and showed that the drug was safe at all dose levels tested $(1,3$ and $10 \mathrm{mg} / \mathrm{kg}$, administered every 2 weeks) without reaching a maximum tolerated dose [24]. Promising antitumor activity was demonstrated across tumor types, which led to a number of trials showing durable responses in melanoma and NSCLC [27-30]. Data on pembrolizumab in GEA are now reported and the main safety and tolerability results of relevant Phase I and 2 trials are summarized in Table 1. It is worth noting that these studies generally excluded patients with active autoimmune disease requiring systemic therapy aside from replacement hormone therapy for endocrinopathies. 
KEYNOTE-012 was the first multicohort trial that included investigation of pembrolizumab in GEA [31]. In this international Phase Ib trial, 39 patients with PD-L1 positive (at least 1\% of scoreable cells using a prototype assay based on neoplastic cells and contiguous mononuclear inflammatory cells - the so-called combined positivity score [CPS]) recurrent or metastatic GEA received pembrolizumab $10 \mathrm{mg} / \mathrm{kg}$ every 2 weeks for up to 24 months. Nineteen (49\%) patients were Asian, 19 (49\%) were white and one (2.6\%) was Native American or Alaska native. Most patients were pretreated, with $85 \%$ having had at least one prior therapy for metastatic disease. Five out of 39 patients (13\%) had grade 3 or 4 treatment-related adverse events (TRAEs) including grade 3 fatigue, grade 3 pemphigoid, grade 3 hypothyroidism, grade 3 peripheral sensory neuropathy and grade 4 pneumonitis. No patients stopped pembrolizumab due to TRAEs. Thirty-six patients were evaluable for response of which eight patients (22\%, 95\% CI: 10-39) had a partial response (PR) on central review. There were no complete responses. Median duration of response was 40 weeks. Data on microsatellite instability were available for 24 patients of which four were MSI-H. Two out of the four patients with MSI-H tumors had a PR while the other two had progressive disease (PD). EBV status was not collected. Although trends were observed between either higher PD-L1 expression on tumor/mononuclear cells or high IFN- $\gamma$ signature and response, these were not statistically significant in the small subset analyses of this pilot study. Median progression-free survival (PFS) was 1.9 months (95\% CI: 1.8-3.5) and median OS was 11.4 months (95\% CI: 5.7-not reached).

KEYNOTE-028 is an ongoing multicohort Phase Ib trial evaluating pembrolizumab in PD-L1 positive advanced solid tumors. In the esophageal carcinoma cohort, 23 patients with PD-L1 positive (expression in tumor or stroma by IHC) EGJ AC or esophageal SCC received second or subsequent-line pembrolizumab $10 \mathrm{mg} / \mathrm{kg}$ every 2 weeks for up to 24 months [32]. Preliminary data indicated that the majority of patients had squamous histology (74\%) and received at least two prior lines of therapy (87\%). Nine out of 23 patients (39\%) had TRAEs and four patients (17\%) had grade 3 TRAEs. No patients died or discontinued the study drug due to TRAEs. The objective response rate (ORR) was 30\% (95\% CI: 13.2-52.9) and the stable disease (SD) rate was $13.0 \%$ (95\% CI: 2.8-33.6). PFS rates at 6 and 12 months were $30 \%$ and $22 \%$, respectively. The relationship between PD-L1 expression levels and degree of antitumor activity has not been reported. It is worth noting that while AC and SCC of the esophagus have significant differences in their risk factors, precursor lesions, biology and response to conventional chemotherapy [33], KEYNOTE-028, as well as some other trials reviewed here, included both of these histologies for trial eligibility. Preliminary data of KEYNOTE-028 did not include subgroup analysis. Results of trials that lump together tumors of various histologies should be interpreted with caution if they are not powered to detect differences in outcomes based on histology. Interestingly, The Cancer Genome Atlas (TCGA) also recently described the genomic profiles of 164 untreated esophageal carcinomas and found that esophageal AC closely resembled the chromosomal instability subtype of gastric AC, suggesting that these two types of malignancies could be grouped together as a single entity across anatomical locations [33]. The molecular features of esophageal SCC resembled SCC from other organs, however did not suggest a causative role for human papillomavirus.

The promising tolerability and responses in KEYNOTE-012 and KEYNOTE-028 led to the development of Phase II trials investigating pembrolizumab alone and in combination with other agents, in the first- and subsequentline settings. In Cohort 1 of KEYNOTE-059, a multicohort international Phase II trial, patients with recurrent or metastatic GEA who had progressed on at least two lines of prior therapy received pembrolizumab $200 \mathrm{mg}$ every 3 weeks [34]. Prospective PD-L1 testing for this and all subsequent pembrolizumab studies defined positivity as the CPS of $\geq 1$ where CPS was the number of PD-L1 staining tumor cells, lymphocytes and macrophages divided by the total number of viable tumor cells multiplied by 100 . Updated results of this 259 patient cohort at a median follow-up of 6 months showed an ORR of 12\% (95\% CI: 8-17) overall; 16\% (95\% CI: 11-23) in PD-L1 positive ( $\geq 1 \%$ of tumor or stromal cells) patients and 6\% (95\% CI: 3-13) in PD-L1 negative patients [35]. Responses were more impressive when looking solely at patients treated in the third-line setting; ORR 21.3\% (95\% CI: 12.7-32.3) with CR rate 4.0\% (95\% CI: 0.8-11.2) in PD-L1 positive patients and ORR 6.9\% (95\% CI: 1.9-16.7) with CR rate 3.4\% (95\% CI: 0.4-11.9) in PD-L1 negative patients [34]. Median PFS was 2 months (95\% CI: 2-2) and median OS 6 months (95\% CI: 4-7) [35]. Forty-six (18\%) patients experienced grade 3-5 TRAEs leading to discontinuation in seven patients (3\%) and death in two patients (1\%).

Cohort 3 of KEYNOTE-059 investigated pembrolizumab $200 \mathrm{mg}$ every 3 weeks in the first-line setting of patients with HER2-negative and PD-L1 positive recurrent or metastatic GEA [36]. Preliminary results of 31 PDL1 positive patients at a median follow-up of 14.5 months showed an ORR of $26 \%$ (95\% CI: 11.9-44.6) with CR in $3.2 \%$ of patients. Median PFS was 3.3 months (95\% CI: 2.0-6.0) and median OS was not reached. Six months and 12 months OS rates were $73 \%$ and $62 \%$ respectively. Twenty-four patients $(77 \%)$ had TRAEs of any grade and 
$7(23 \%)$ had grade 3-5 events. One patient died due to treatment related pneumonitis. Updated data at a median follow-up of 18 months showed unchanged ORR, median PFS, median OS and rate of $\geq$ grade 3 TRAEs [35].

\section{Combination therapy with pembrolizumab}

Cohort 2 of KEYNOTE-059 enrolled 25 patients with HER2-negative recurrent or metastatic GEA to receive firstline therapy with pembrolizumab $200 \mathrm{mg}$ every 3 weeks, cisplatin $80 \mathrm{mg} / \mathrm{m}^{2}$ for six cycles and $5 \mathrm{FU} 800 \mathrm{mg} / \mathrm{m}^{2}$ (or capecitabine $1000 \mathrm{mg} / \mathrm{m}^{2}$ ) every 3 weeks [37]. Preliminary results of 25 patients showed that at a median duration of follow-up of 14 months, overall ORR was 60\% (95\% CI: 39-79); ORR was 73\% (95\% CI: 45-92) in 15 PD-L1 positive ( $\geq 1 \%$ of tumor or stromal cells) patients and $38 \%$ (95\% CI: 9-76) in 8 PD-L1 negative patients. Median PFS was 7 months (95\% CI: 6-11) and median OS was 14 months (95\% CI: 9-not estimable). Nineteen (76\%) of patients experienced $\geq$ grade 3 TRAEs and TRAEs of any grade led to discontinuation in 3 patients [35].

As previously discussed, the VEGFR-2 inhibitor ramucirumab was shown to confer a survival benefit in the second-line setting of advanced GEA [9,10]. In an ongoing multicohort Phase Ia/b trial of patients with advanced GEA who have progressed on prior systemic therapy, ramucirumab at two different dose levels was combined with pembrolizumab $200 \mathrm{mg}$ every 3 weeks in 40 patients [38]. Preliminary results generated no new safety signals and demonstrated that the combination regimen is potentially active. Any grade TRAEs occurred in 31 patients (78\%) and ten patients (25\%) experienced grade 3-4 TRAEs. One treatment related death occurred due to pneumocystis pneumonia and pulmonary sepsis. Partial responses were seen in 7.5\% (3 of 40 patients) and disease control in 45\%. Median PFS was 2.1 months for patients given pembrolizumab in combination with ramucirumab $8 \mathrm{mg} / \mathrm{kg}$ on day 1 and day 8 . Median PFS was 2.6 months for patients given pembrolizumab plus ramucirumab $10 \mathrm{mg} / \mathrm{kg}$ on day 1 (Table 1).

\section{Ongoing Phase II \& III trials \\ First line}

KEYNOTE-062 is a randomized Phase III trial comparing pembrolizumab $200 \mathrm{mg}$ every 3 weeks alone versus pembrolizumab in combination with chemotherapy (cisplatin $80 \mathrm{mg} / \mathrm{m}^{2}$ every 3 weeks and a fluoropyrimidine $-5 \mathrm{FU} 800 \mathrm{mg} / \mathrm{m}^{2}$ on days $1-5$ every 3 weeks or capecitabine $1000 \mathrm{mg} / \mathrm{m}^{2}$ twice daily on days $1-14$ of each 3 week cycle) versus chemotherapy plus placebo in first-line therapy for PD-L1 positive and HER2negative advanced GEA [39]. Enrollment of approximately 750 patients is completed and results are eagerly awaited (NCT02494583). KEYNOTE-590 is a randomized Phase III trial comparing pembrolizumab $200 \mathrm{mg}$ every 3 weeks plus cisplatin/5FU (cisplatin $80 \mathrm{mg} / \mathrm{m}^{2}$ every 3 weeks, $5 \mathrm{FU} 800 \mathrm{mg} / \mathrm{m}^{2} /$ day continuous infusion days 1-5) versus placebo plus cisplatin/5FU in advanced esophageal SCC and Siewert type 1 EGJ AC regardless of PD-L1 status (NCT03189719) [40]. All ongoing pembrolizumab studies in GEA are summarized in Table 2.

There are two ongoing exploratory trials investigating the combination of pembrolizumab, trastuzumab, capecitabine and cisplatin in the first-line setting. An American trial is enrolling 37 patients with HER2-positive GEA (NCT02954536) and a Korean trial is recruiting 49 patients with HER2-positive GC (NCT02901301) to receive this combination $[41,42]$.

\section{Second line}

KEYNOTE-061 is a randomized, open-label, Phase III trial comparing pembrolizumab to paclitaxel in the secondline treatment of advanced GEA that has progressed after first-line treatment with fluoropyrimidine and platinum doublet chemotherapy [43]. Patients are randomized 1:1 to pembrolizumab $200 \mathrm{mg}$ every 3 weeks versus paclitaxel $80 \mathrm{mg} / \mathrm{m}^{2}$ iv. on day 1,8 and 15 of a 28 day cycle until progression, toxicity, or completion of 24 months therapy (pembrolizumab arm only). Enrollment is complete with 592 patients (NCT02370498). The primary end points are PFS and OS in PD-L1 positive patients, and secondary end points include outcomes of PD-L1 negative patients. KEYNOTE-181 is a randomized, open-label, Phase III trial comparing pembrolizumab $200 \mathrm{mg}$ every 3 weeks to investigator's choice chemotherapy (docetaxel $75 \mathrm{mg} / \mathrm{m}^{2}$ every 3 weeks, irinotecan $180 \mathrm{mg} / \mathrm{m}^{2}$ every 2 weeks, or paclitaxel $80-100 \mathrm{mg} / \mathrm{m}^{2}$ on days 1,8 and 15 every 4 weeks) in the second-line treatment of advanced esophageal SCC and Siewert type 1 EGJ AC regardless of PD-L1 status (NCT02564263) [44]. Six hundred patients are expected to enroll.

As discussed above, trastuzumab is not approved for advanced HER2-positive GEA beyond progression in first-line therapy [7]. However, in preliminary Phase I data, the anti-HER2 monoclonal antibody margetuximab demonstrated promising anti-tumor activity in patients with HER2 positive GC [45]. A Phase Ib/II dose-escalation 


\begin{tabular}{|c|c|c|c|}
\hline Study & Study design & Primary end points & Ref. \\
\hline $\begin{array}{l}\text { NCT02494583 } \\
\text { KEYNOTE-062 }\end{array}$ & $\begin{array}{l}\text { Phase III, PEM } 200 \mathrm{mg} \text { q } 3 \mathrm{w} \text { vs PEM/chemo vs placebo/chemo } \\
\text { Multicenter, international } \\
\text { Estimated } \mathrm{n}=750 \text { (GEA, PD-L1+) } \\
1 \mathrm{~L} \text { therapy }\end{array}$ & PFS and OS & [39] \\
\hline $\begin{array}{l}\text { NCT03189719 } \\
\text { KEYNOTE-590 }\end{array}$ & $\begin{array}{l}\text { Phase III, PEM } 200 \mathrm{mg} \text { q3} w+\mathrm{CIS} / 5 \mathrm{FU} \text { vs placebo/CIS } / 5 \mathrm{FU} \\
\text { Multicenter, international } \\
\text { Estimated } \mathrm{n}=700 \text { (E SCC, Siewert type } 1 \mathrm{EGJ} \mathrm{AC)} \\
1 \mathrm{~L} \text { therapy }\end{array}$ & PFS, OS & [40] \\
\hline NCT02954536 & $\begin{array}{l}\text { Phase II, PEM } 200 \mathrm{mg} \mathrm{q} 3 \mathrm{w}+\text { TRAS }+ \text { CIS } / \text { CAPE } \\
\text { Multicenter, US } \\
\text { Estimated } n=37 \text { (GEA, HER2 }+ \text { ) } \\
1 \mathrm{~L} \text { therapy }\end{array}$ & PFS & [41] \\
\hline NCT02901301 & $\begin{array}{l}\text { Phase } \mathrm{Ib} / 2, \text { PEM } 200 \mathrm{mg} \text { q } 3 \mathrm{w}+\text { TRAS }+ \text { CIS } / \text { CAPE } \\
\text { Korea } \\
\text { Estimated } \mathrm{n}=49(\mathrm{G} \mathrm{AC}, \mathrm{HER} 2+) \\
1 \mathrm{~L} \text { therapy }\end{array}$ & $\begin{array}{l}\text { Recommended dose } \\
\text { ORR }\end{array}$ & [42] \\
\hline $\begin{array}{l}\text { NCT02370498 } \\
\text { KEYNOTE-061 }\end{array}$ & $\begin{array}{l}\text { Phase III, PEM } 200 \mathrm{mg} q 3 \mathrm{w} \text { vs paclitaxel } \\
\text { Estimated } \mathrm{n}=592(\mathrm{GEA}) \\
2 \mathrm{~L} \text { therapy }\end{array}$ & PFS and OS in PD-L1+ & [43] \\
\hline $\begin{array}{l}\text { NCT02564263 } \\
\text { KEYNOTE-181 }\end{array}$ & $\begin{array}{l}\text { Phase III, PEM } 200 \text { mg q3} w \text { vs chemotherapy } \\
\text { Multicenter, international } \\
\text { Estimated } n=600 \text { (E SCC, Siewert type } 1 \text { EGJ AC) } \\
2 \text { L therapy }\end{array}$ & OS & [44] \\
\hline NCT02689284 & $\begin{array}{l}\text { Phase } \mathrm{lb} / 2, \mathrm{PEM} 200 \mathrm{mg} \mathrm{q} 3 \mathrm{w}+\text { margetuximab } \\
\text { Multicenter, international } \\
\text { Estimated } \mathrm{n}=72(\mathrm{GEA}, \mathrm{HER} 2+) \\
2 \mathrm{~L} \text { therapy }\end{array}$ & $\begin{array}{l}\text { Recommended dose } \\
\text { Duration of response } \\
\text { ORR }\end{array}$ & [46] \\
\hline $\begin{array}{l}\text { NCT02559687 } \\
\text { KEYNOTE-180 }\end{array}$ & $\begin{array}{l}\text { Phase II, PEM } 200 \text { mg q3w } \\
\text { Multicenter, international } \\
\text { Estimated } n=100 \text { (E SCC, Siewert type } 1 \text { EGJ AC) } \\
3 \text { L therapy }\end{array}$ & ORR & [47] \\
\hline NCT02830594 & $\begin{array}{l}\text { Phase II, PEM } 200 \text { mg q3w + RT } 30 \text { Gy } \\
\text { US } \\
\text { Estimated } n=14 \text { (E SCC, GEA) } \\
\text { Any line of therapy }\end{array}$ & $\begin{array}{l}\text { Biomarkers of immune response } \\
\text { ORR }\end{array}$ & [49] \\
\hline NCT02563548 & $\begin{array}{l}\text { Phase Ib PEM + PEGPH } 20 \\
\text { Multicenter, US } \\
\text { Estimated } n=81 \text { (G AC, NSCLC, hyaluronan-high) } \\
\text { Up to } 3 \text { L therapy for G AC }\end{array}$ & ORR & [50] \\
\hline NCT03122548 & $\begin{array}{l}\text { Phase II, PEM } 200 \text { mg q3} w+\text { CRS-207 } \\
\text { Multicenter, US } \\
\text { Estimated } n=79 \text { (GEA) } \\
3 \text { L therapy }\end{array}$ & Number of patients with TRAEs & [51] \\
\hline \multicolumn{4}{|c|}{$\begin{array}{l}1 \text { L: First line; } 2 \text { L: Second line; } 3 \text { L: Third line; AC: Adenocarcinoma; CAPE: capecitabine; CIS: cisplatin; E: Esophageal; EGJ: Esophagogastric junction; G: Gastric; GEA: Gastric and } \\
\text { esophagogastric adenocarcinoma; NSCLC: Non-small-cell lung cancer; OS: Overall survival; PEM: Pembrolizumab; PFS: Progression free survival; q3w: Every } 3 \text { week; RT: Radiation } \\
\text { therapy; SCC: Squamous cell carcinoma; TRAE: Treatment related adverse event; TRAS: Trastuzumab; US: United States. }\end{array}$} \\
\hline
\end{tabular}

and expansion study of margetuximab plus pembrolizumab in the second and subsequent-line setting of patients with HER2-positive GEA who have progressed past first line trastuzumab plus chemotherapy is currently enrolling (NCT02689284) [46].

Third line \& beyond

KEYNOTE-180 is a single-arm global Phase II trial being conducted in patients with advanced/metastatic SCC of the esophagus or Siewert type $1 \mathrm{AC}$ of the EGJ who have progressed on at least two prior lines of therapy (NCT02559687) [47]. Approximately 100 patients will receive pembrolizumab $200 \mathrm{mg}$ every 3 weeks for up to 2 years. The study is ongoing and results are awaited.

It is hypothesized that radiation increases tumor PD-L1 expression, and thus adding pembrolizumab to radiation is currently being investigated in several tumor types. Combining PD-L1 blockade with radiation therapy has been shown to synergistically enhance host antitumor immunity in preclinical models [48]. This synergy is theorized to induce an abscopal effect, which refers to the phenomenon of non-irradiated lesions regressing after radiation to a different tumor site. Pembrolizumab $200 \mathrm{mg}$ every 3 weeks plus palliative radiation therapy of 30 Gy over 10 
fractions is currently being investigated in a Phase II trial of patients with metastatic SCC of the esophagus or GEA (NCT02830594) [49].

PEGPH20, an investigational agent that enzymatically removes hyaluronan from the tumor microenvironment, is being studied in combination with pembrolizumab in the third-line setting in hyaluronan-high advanced GC (NCT02563548) [50].

CRS-207, an engineered live-attenuated strain of Listeria monocytogenes expressing the tumor-associated antigen mesothelin, combined with pembrolizumab in the third-line setting is also being investigated in a Phase II trial of advanced GEA (NCT03122548) [51]. These ongoing trials are summarized in Table 2.

\section{Biomarkers for responsiveness to PD-1 blockade}

GEA is a heterogeneous group of diseases with variable benefit from pembrolizumab. The identification of biomarkers that predict response to immunotherapy may help guide selection of patients that are most likely to benefit from PD-1 blockade. PD-1 and PD-L1 staining has been studied as predictive response biomarkers in several tumor types. In advanced NSCLC, PD-L1 expression of at least $50 \%$ of tumor cells is correlated with prolonged PFS and OS with pembrolizumab treatment [30]. However, in KEYNOTE-012, although a trend was observed, there was no statistically significant association between higher PD-L1 expression on tumor cells and response [31]. Of note, all patients in this trial were PD-L1 positive (at least $1 \%$ of scoreable cells using a prototype assay) so that particular study cannot ascertain differential benefit of pembrolizumab in PD-L1 positive versus negative tumors using this antibody and cut-off [31,52]. This is also the case for KEYNOTE-059 Cohort 3, KEYNOTE-062 and all other studies limiting enrollment to only patients with PD-L1 positive tumors. In preliminary data from Cohorts 1 and 2 of KEYNOTE-059, PD-L1 positive ( $\geq 1 \%$ tumor and/or stromal cells) patients had a higher ORR compared with PD-L1 negative patients [34,37]. At this time, current available data suggest that PD-L1 testing can enrich for GEA patients who are more likely to respond to pembrolizumab, with a $21.3 \%$ response rate, seen in third-line PD-L1 positive patients from KEYNOTE-059 Cohort 1 . However, there is room for improvement to better predict which PD-L1 positive patients are most likely to respond to therapy. Conversely, a PD-L1 negative result, having a $6.8 \%$ response rate in the third-line setting in KEYNOTE-059 Cohort 1 , does have a high negative predictive value. These PD-L1 negative patients may be better served on studies combining pembrolizumab with other therapies such as cytotoxics, targeted therapies, tumor stroma modulators and/or other checkpoint inhibitors. Data regarding predictive value of PD-L1 expression on tumor and stromal elements continues to be collected to further define PD-L1 testing as a predictive biomarker of benefit to pembrolizumab. Of note, the definition of PD-L1 positivity is not standardized and can vary based on reagent used, types of cells assessed, and scoring systems [52,53]. Studies investigating pembrolizumab utilize a pharmDx immunohistochemistry (IHC) assay (PD-L1 IHC 22C3; Agilent Technologies, CA, USA) and, as previously described, determine PD-L1 positivity based on the CPS (assessment of both tumor cells and tumor-infiltrating lymphocytes [TILs]) [54]. In contrast, studies investigating nivolumab use a different pharmDx IHC assay (PD-L1 IHC 28-8; Agilent Technologies) that relies on assessment of tumor cells only to determine PD-L1 positivity. The CPS score may be better able to predict response to therapy given that it incorporates assessment of the tumor microenvironment in addition to actual tumor cells.

The Cancer Genome Atlas research network recently reported the molecular characteristics of 295 primary untreated gastric ACs as part of The Cancer Genome Atlas project [55]. GC was classified into the following four subtypes based on integrated molecular profiling: MSI tumors (22\%), EBV+ tumors (9\%), tumors exhibiting chromosomal instability (50\%) and genomically stable tumors (20\%). MSI has also been reported in $6.6 \%$ of untreated resected Barrett esophagus-associated EGJ AC [56]. The subsequent TCGA esophagus analysis of 164 localized esophageal cancers including both AC and SCC demonstrated a lower incidence of MSI-H at 4\% compared with distal GC, and all occurring in type II and III EGJ tumors. It is thus recognized that MSI-H incidence is higher in distal GC compared with proximal EGJ tumors [33,55]. A limitation of these TCGA datasets is the lack of applicability of incidence rates to the stage IV setting. MSI-H tumors may have a relatively good prognosis in the perioperative setting and therefore do not recur as frequently as microsatellite stable (MSS) tumors [57]. Further support for this comes from a recent secondary post hoc analysis of the Medical Research Council Adjuvant Gastric Infusional Chemotherapy trial that assessed MSI status of 303 patients with resectable GEA treated with surgery alone versus perioperative chemotherapy plus surgery [58]. Twenty of 303 patients (6.6\%) with locally advanced disease had MSI-H tumors. When compared with patients who had MSS/MSI-low tumors, MSI-H patients had superior survival when treated with surgery alone and inferior survival when treated with perioperative chemotherapy plus surgery, reminiscent of observations from perioperative colorectal cancer studies [59]. As such, 
the incidence of MSI-H in advanced metastatic GC, although not well reported to date, is likely less than 5\% and even less for EGJ cancers, as seen in our relatively large unpublished stage IV cohort.

The relevance of MSI-H tumors, and EBV+ tumors which have similarly low rates and anatomical predilection within the gastric fundus/body [33,55], is that both are thought to be more likely sensitive to PD-1 blockade. This is due to high neoantigen levels in MSI-H tumors and due to high degree of PD-L1 positive T-cell infiltration in both MSI-H and EBV+ tumors. Microsatellites are repeats of the same base or sequence of bases found throughout coding and noncoding regions of the genome. DNA polymerases have a tendency to make errors in these regions by either inserting additional bases on the synthesized strand or by removing them on the template strand, resulting in mismatched DNA strands [60]. After replication of DNA, mismatch repair (MMR) machinery glides along DNA and fixes mismatches. However, certain tumors have defects in the DNA MMR system (MMR deficient, dMMR) resulting in 100-1000 times increased rate of frameshift and missense mutations [60]. These tumors are labeled as having high levels of microsatellite instability. MMR deficient tumors are thought to produce a greater number of mutation-associated neoantigens and have a higher likelihood of antitumor response [61]. Across tumor types, patients with dMMR tumors are more likely to respond to PD-1 blockade than MMR proficient tumors [61]. KEYNOTE-158 enrolled non-colorectal cancer (CRC) MSI-H patients who received at least one prior therapy to receive pembrolizumab $200 \mathrm{mg}$ every 3 weeks [62]. Two patients with GC were enrolled in this Phase II trial. The ORR for chemorefractory MSI-H non-CRC was an impressive 43\% (95\% CI: 21.8-66.0). Based on these data, in an unprecedented manner, pembrolizumab was FDA-approved for patients with unresectable or metastatic MSI-H or dMMR of any solid tumor type, including GEA, that progressed following prior treatment and who have no satisfactory alternatives [63]. Again, for stage IV GEA, this will affect relatively few patients, but when present, is a very important treatment option.

EBV is a human herpes virus implicated in a variety of malignancies including lymphoma, nasopharyngeal carcinoma and gastric AC. Patients with EBV+ GC have improved survival which has been in part attributed to the associated rich $\mathrm{CD}^{+}{ }^{+} \mathrm{T}$-cell infiltrate present as part of the immune response against EBV [22,64,65]. EBV+ GC have increased PD-L1 and PD-L2 expression [55]. PD-L1 staining, depending on the antibody and assay used, has been reported in 50\% of tumor cells and $94 \%$ of immune cells in EBV+ GC [22]. In contrast, amongst EBV negative GC, PD-L1 staining has been reported in 10\% of tumor cells (of which all were MSI-H) and 39\% of immune cells [22]. Again, given the increased PD-L1/PD-L2 expression on tumor cells and TILs in EBV+ GC, this subtype may be particularly susceptible to PD-1 blockade, although to date there are no reports detailing checkpoint blockade response in EBV+ patients.

Gene expression profiling of tumors can identify signatures that may predict response to immunotherapy. Specifically, interferon-related genes are being studied given their role in PD-L1 regulation. Upon encountering neoplastic antigens, activated T cells secrete interferons that induce expression of PD-L1 on neoplastic or stromal cells which in turn inhibit the antitumor immune response [66]. This phenomenon known as adaptive immune resistance allows tumors to evade destruction [66]. An interferon-inflammatory immune gene signature is associated with response to pembrolizumab in advanced melanoma [67]. In KEYNOTE-012, a six-gene signature of IFN- $\gamma$ related genes (CXCL9, CXCL10, IDO1, IFNG, HLA-DRA and STAT1) was analyzed to assess its association with response to pembrolizumab in GEA [31]. While there were trends toward association between IFN- $\gamma$ signature score and ORR and PFS, these values were not statistically significant. However, gene expression data were only evaluable in 30 samples and a signal may still be detected in larger studies. EBV+ and MSI-H GCs have enrichment of an IFN- $\gamma$ immune response signature, which appears to reflect their amenability to immune checkpoint blockade [22].

Tumor mutation burden (TMB) quantifies mutations present in tumor cells and is currently under investigation as a potential immunotherapy biomarker. In patients with stage IV NSCLC, an exploratory analysis found patients with high TMB had a greater response rate and longer PFS compared with low/medium TMB, when treated with nivolumab versus platinum-based chemotherapy [68]. There was no association between level of TMB and level of tumor PD-L1 expression, however patients with PD-L1 expressions of $<1 \%$ were excluded from this trial. It was recently reported that $7-12 \%$ of GCs have defective double-strand DNA break repair by homologous recombination [69]. Given that defective double-strand break repair increases TMB, this subset of patients may be more responsive to checkpoint blockade. However, additional studies are needed to clarify the role of TMB in GEA.

Finally, the role of the tumor microenvironment in guiding immunotherapy is an evolving area of research. TILs are a component of the host immune response against cancer cells. In patients with resected GCs, density of $\mathrm{CD}^{+}$TILs is prognostic of improved survival [70]. In resected EBV associated GCs, stromal TIL positivity is 
associated with longer recurrence-free survival and disease-free survival [71]. As above, patients with MSI-H tumors and associated TILs have better prognosis after surgical resection, particularly without receiving perioperative chemotherapy [56,58]. In patients with dMMR metastatic GC treated with chemotherapy, the presence of TILs was also linked to improved survival [72]. In contrast, a retrospective study of resected GEA showed that increased CD8 ${ }^{+}$ T-cell infiltration in tumor and stroma was correlated with increased PD-L1 expression and impaired PFS and OS, suggesting an adaptive immune response mechanism [73]. There are contradictory reports on the correlation between $\mathrm{CD}^{+}$or FOXP3 ${ }^{+}$lymphocytes with OS [74]. Additional research is needed to clarify whether TILs may be prognostic in the perioperative setting and if this differs in the metastatic setting and by MSI and EBV status. Similarly, further studies are needed to confirm/refute whether TILs are predictive of response to immunotherapy in GEA, and whether TIL-rich tumors such as MSI-H and EBV positive tumors differ from TIL-rich MSS and EBV negative tumors. From a biomarker assay perspective, the CPS PD-L1 score currently appears to best predict benefit from these checkpoint inhibitors, very likely due to its ability to quantify both tumor PD-L1 expression as well as TIL PD-L1 expression, in essence assessing TIL density.

\section{Other checkpoint inhibitors in GEA}

There are a number of other monoclonal antibody checkpoint inhibitors that are being investigated in GEA. These include nivolumab (anti-PD-1), avelumab (anti-PD-L1), durvalumab (anti-PD-L1), atezolizumab (anti-PD-L1), ipilimumab (anti-CTLA-4) and tremelimumab (anti-CTLA4). Preliminary Phase I/II data among heavily pretreated patients with advanced or metastatic solid tumors (including GC) have shown promising antitumor activity with nivolumab alone or in combination with ipilimumab [75]. A randomized Phase III trial, ATTRACTION-02, investigated third or later line nivolumab versus placebo in advanced GEA, regardless of PD-L1 status, and was recently reported [76]. At the time of preliminary analysis, median OS was improved with nivolumab at 5.3 months compared with placebo at 4.1 months (HR: 0.63 ; 95\% CI: 0.50-0.78; $\mathrm{p}<0.0001$ ). The ORR was $11 \%$ (95\% CI: 7.7-15.6) with nivolumab compared with $0 \%$ (95\% CI: $0.0-2.8)$ with placebo $(\mathrm{p}<0.0001)$. This response rate was quite similar to that of all Cohort 1 patients enrolled irrespective of PD-L1 status in the KEYNOTE-059 study [34]. Median PFS was 1.6 months with nivolumab compared with 1.5 months with placebo (HR: 0.60; 95\% CI: $0.49-0.75 ; \mathrm{p}<00001)$. There were $12 \% \geq$ grade 3 TRAEs with nivolumab and $2.7 \%$ discontinued study treatment due to any grade TRAE, compared with 5.5 and $2.5 \%$ respectively with placebo. Recognizing the limitations of cross-trial comparisons, differences in PD-L1 biomarker assays (antibody and scoring system) [52], as well as nonrandomized Phase IIa studies, nivolumab and pembrolizumab appear to have a similar ORR in all comers irrespective of PD-L1 status, as well as rate of $\geq$ grade 3 TRAEs in the salvage treatment setting of GEA. Updated results were reported by PD-L1 status in only $40 \%$ of patients enrolled having adequate and available samples [77]. This again was performed using the different companion pharmDx IHC assay (PD-L1 IHC 28-8; Agilent Technologies) and scoring of only tumor cells, not like the CPS scoring. The authors reported no significant differences in response rates or median OS by PD-L1 status. In stark contrast to studies with pembrolizumab and its CPS assay, this again may be due to the inability to identify high TIL density tumors that would be considered positive by the CPS assay, while considered negative with the non-CPS scoring assay. Further prospective studies comparing the different antibodies and scoring systems are needed to clarify which approach is best to accurately predict benefit from these checkpoint inhibitors. Other initial Phase I data have suggested activity of avelumab and durvalumab in GC, however more robust Phase II and Phase III data are still awaited [78,79].

\section{Regulatory affairs}

In September 2017, the FDA approved pembrolizumab for third-line treatment of recurrent locally advanced or metastatic PD-L1-positive GEA that has progressed after two prior lines of chemotherapy including 5FU- and platinum-based therapy as well as targeted therapy against HER2 as appropriate [21]. Pembrolizumab is already approved in both the US and European Union (EU) for unresectable or metastatic melanoma, metastatic NSCLC with no EGFR or anaplastic lymphoma kinase (ALK) genomic tumor aberrations (first-line therapy in tumors with PD-L1 >50\%), and metastatic NSCLC after disease progression with prior chemotherapy (in tumors with $>1 \%$ PD-L1 expression, tumors with EGFR and ALK positivity should also have received targeted therapy) [20,80]. In addition, pembrolizumab is approved in the US and EU for classical Hodgkin lymphoma with slightly different stipulations (in the US for patients who have relapsed after $\geq 3$ lines of therapy and in the EU for patients who have failed autologous stem cell transplant and brentuximab vedotin or who are transplant ineligible and have failed brentuximab vedotin) [20,80]. Finally, pembrolizumab is also approved in the US for the following additional 
indications: metastatic nonsquamous NSCLC with no EGFR or ALK genomic tumor aberrations in combination with pemetrexed and carboplatin as first-line therapy, recurrent or metastatic head and neck SCC with disease progression with platinum-containing chemotherapy, locally advanced or metastatic urothelial carcinoma when cisplatin-containing chemotherapy is not an option and unresectable or metastatic MSI-H or dMMR cancer that has progressed following prior treatment and with no alternative options [20].

\section{Conclusion}

Advanced GEA is an aggressive disease with poor outcomes despite chemotherapy. The development of checkpoint blockade has led to a paradigm shift in cancer treatment. Pembrolizumab is currently approved in the US for thirdline treatment of advanced PD-L1 positive GEA and for second-line treatment of advanced MSI-H or dMMR GEA. Pembrolizumab has a manageable safety profile and robust antitumor activity in first- and subsequent-line management of GEA, when used either as monotherapy or in combination with other agents. Phase III trials are now underway to assess the efficacy of pembrolizumab in the first- and second-line settings of advanced disease.

\section{Executive summary}

Pembrolizumab pharmacokinetic properties

- Pembrolizumab is a humanized monoclonal lgG4 kappa antibody that blocks PD-1 binding with PD-L1 and PD-L2.

- Bodyweight, age, sex, tumor type, tumor burden, renal impairment and hepatic impairment have no clinically relevant impact on pembrolizumab exposure.

Phase I evidence for pembrolizumab in gastroesophageal adenocarcinoma

- In patients with advanced PD-L1+ gastroesophageal adenocarcinoma (GEA), pembrolizumab is well tolerated with a $13 \%$ rate of $\geq$ grade 3 treatment-related adverse events (TRAEs) and a $22 \%$ objective response rate (ORR). $85 \%$ of these patients had received at least one prior therapy for metastatic disease.

- In patients with advanced PD-L1+ esophageal or esophagogastric junction cancer, second- or subsequent-line pembrolizumab has a $17 \%$ rate of $\geq$ grade 3 TRAEs and a $30 \%$ ORR.

- In patients with advanced GEA, second- and subsequent-line therapy with combination pembrolizumab plus ramucirumab has generated no new safety signals, a 78\% rate of any grade TRAEs and $7.5 \%$ ORR in patients with any PD-L1 status.

Phase Il evidence for pembrolizumab in GEA

- In patients with advanced GEA, third- and subsequent-line pembrolizumab has a $18 \%$ rate of $\geq$ grade 3 TRAEs, $12 \%$ ORR (16\% in PD-L1+ patients and 6\% in PD-L1-negative patients), 2 months median progression-free survival (PFS) and 6 months overall survival (OS), in a 259-patient study which was the basis for the recent approval of pembrolizumab for PD-L1+ patients.

- In patients with advanced HER2-negative and PD-L1+ GEA, first-line pembrolizumab monotherapy has a $23 \%$ rate of $\geq$ grade 3 TRAEs, $26 \%$ ORR, 12 month OS rate of $62 \%$ and median PFS of 3.3 months in an exploratory 31 patient study, being further assessed in a Phase III study.

- In patients with advanced HER2-negative GEA, first-line therapy with combination pembrolizumab, cisplatin and fluorouracil (or capecitabine) has a $76 \%$ rate of $\geq$ grade 3 TRAEs, $60 \%$ ORR ( $73 \%$ in PD-L1+ patients and $38 \%$ in PD-L1 negative patients), median PFS 7 months and median OS 14 months in an exploratory 25 patient study, which will be further assessed in a Phase III study.

Discussion

- There are several ongoing studies including four Phase III trials of pembrolizumab in the first- or second-line settings and earlier phase studies combining pembrolizumab with other therapies.

- Epstein-Barr virus positivity, microsatellite instability, interferon gene signature, tumor mutation burden and PD-L1 expression are all being studied as potential biomarkers to predict response to immunotherapy.

Pembrolizumab is now a standard of care therapy for microsatellite instability-high tumors including GEA.

- There are a number of other checkpoint inhibitors being investigated in GEA of which nivolumab has the largest cohorts and most robust evidence for safety and efficacy reported to date.

- Pembrolizumab is US FDA approved for treatment of recurrent locally advanced or metastatic PD-L1+ GEA that has progressed on at least two prior lines of chemotherapy including fluorouracil- and platinum-based therapy, and anti-HER2 therapy as appropriate. Pembrolizumab is also approved for second-line treatment of unresectable or metastatic microsatellite instability high or mismatch repair deficient GEA.

Conclusion

- Pembrolizumab has a manageable safety profile and promising antitumor activity in various lines of management of GEA, when used either as monotherapy or in combination with other agents. 
Financial \& competing interests disclosure

Conflicts of interest pertaining to D Cantenacci: Merck, Bristol-Myers Squibb, Lilly, Amgen, Genentech, Taiho Honoraria, Roche Honoraria, Foundation Medicine Honoraria and Guardant Health Honoraria. D Catenacci is funded by the NIH, USA (NIH K23 CA178203-01A1). The authors have no other relevant affiliations or financial involvement with any organization or entity with a financial interest in or financial conflict with the subject matter or materials discussed in the manuscript apart from those disclosed.

No writing assistance was utilized in the production of this manuscript.

\section{References}

Papers of special note have been highlighted as: • of interest; $\bullet \bullet$ of considerable interest

1 Torre LA, Bray F, Siegel RL, Ferlay J, Lortet-Tieulent J, Jemal A. Global Cancer Statistics, 2012. CA Cancer J. Clin. 65(2), 87-108 (2015).

2 Siegel RL, Miller KD, Jemal A. Cancer Statistics, 2017. CA Cancer J. Clin. 67(1), 7-30 (2017).

3 Kamangar F, Dores GM, Anderson WF. Patterns of cancer incidence, mortality, and prevalence across five continents: defining priorities to reduce cancer disparities in different geographic regions of the world. J. Clin. Oncol. 24(14), 2137-2150 (2006).

4 Sehdev A, Catenacci DV. Gastroesophageal cancer: focus on epidemiology, classification, and staging. Discov. Med. 16(87), 103-111 (2013).

5 Al-Batran S-E, Homann N, Schmalenberg H et al. Perioperative chemotherapy with docetaxel, oxaliplatin, and fluorouracil/leucovorin (FLOT) versus epirubicin, cisplatin, and fluorouracil or capecitabine (ECF/ECX) for resectable gastric or gastroesophageal junction (GEJ) adenocarcinoma (FLOT4-AIO): a multicenter, randomized Phase 3 trial. J. Clin. Oncol. 35(15 Suppl.), 4004-4004 (2017).

6 Van Cutsem E, Sagaert X, Topal B, Haustermans K, Prenen H. Gastric cancer. Lancet 388(10060), 2654-2664 (2016).

7 Maron SB, Catenacci DVT. Update on gastroesophageal adenocarcinoma targeted therapies. Hematol. Oncol. Clin. North Am. 31(3), 511-527 (2017).

8 Bang YJ, Van Cutsem E, Feyereislova A et al. Trastuzumab in combination with chemotherapy versus chemotherapy alone for treatment of HER2-positive advanced gastric or gastro-oesophageal junction cancer (ToGA): a Phase 3, open-label, randomised controlled trial. Lancet 376(9742), 687-697 (2010).

9 Fuchs CS, Tomasek J, Yong CJ et al. Ramucirumab monotherapy for previously treated advanced gastric or gastro-oesophageal junction adenocarcinoma (REGARD): an international, randomised, multicentre, placebo-controlled, Phase 3 trial. Lancet 383(9911), 31-39 (2014).

10 Wilke H, Muro K, Van Cutsem E et al. Ramucirumab plus paclitaxel versus placebo plus paclitaxel in patients with previously treated advanced gastric or gastro-oesophageal junction adenocarcinoma (RAINBOW): a double-blind, randomised Phase 3 trial. Lancet Oncol. 15(11), 1224-1235 (2014).

11 Satoh T, Xu RH, Chung HC et al. Lapatinib plus paclitaxel versus paclitaxel alone in the second-line treatment of HER2-amplified advanced gastric cancer in Asian populations: TyTAN - a randomized, Phase III study. J. Clin. Oncol. 32(19), 2039-2049 (2014).

12 Hecht JR, Bang Y-J, Qin SK et al. Lapatinib in combination with capecitabine plus oxaliplatin in human epidermal growth factor receptor 2-positive advanced or metastatic gastric, esophageal, or gastroesophageal adenocarcinoma: TRIO-013/LOGiC - a randomized Phase III trial. J. Clin. Oncol. 34(5), 443-451 (2016).

13 Thuss-Patience PC, Shah MA, Ohtsu A et al. Trastuzumab emtansine versus taxane use for previously treated HER2-positive locally advanced or metastatic gastric or gastro-oesophageal junction adenocarcinoma (GATSBY): an international randomised, open-label, adaptive, Phase 2/3 study. Lancet Oncol. 18(5), 640-653

14 Tabernero J, Hoff PM, Shen L et al. 616OPertuzumab (P) + trastuzumab (H) + chemotherapy (CT) for HER2-positive metastatic gastric or gastro-oesophageal junction cancer (mGC/GEJC): final analysis of a Phase III study (JACOB). Ann. Oncol. 28(Suppl. 5), $\operatorname{mdx} 369$ (2017).

15 Okines AF, Ashley SE, Cunningham D et al. Epirubicin, oxaliplatin, and capecitabine with or without panitumumab for advanced esophagogastric cancer: dose-finding study for the prospective multicenter, randomized, Phase II/III REAL-3 trial. J. Clin. Oncol. 28(25), 3945-3950 (2010).

16 Lordick F, Kang YK, Chung HC et al. Capecitabine and cisplatin with or without cetuximab for patients with previously untreated advanced gastric cancer (EXPAND): a randomised, open-label Phase 3 trial. Lancet Oncol. 14(6), 490-499 (2013).

17 Dutton SJ, Ferry DR, Blazeby JM et al. Gefitinib for oesophageal cancer progressing after chemotherapy (COG): a Phase 3, multicentre, double-blind, placebo-controlled randomised trial. Lancet Oncol. 15(8), 894-904 (2014).

18 Catenacci DVT, Tebbutt NC, Davidenko I et al. Rilotumumab plus epirubicin, cisplatin, and capecitabine as first-line therapy in advanced MET-positive gastric or gastro-oesophageal junction cancer (RILOMET-1): a randomised, double-blind, placebo-controlled, Phase 3 trial. Lancet Oncol. 18(11), 1467-1482 2017).

19 Shah MA, Bang Y, Lordick F et al. Effect of fluorouracil, leucovorin, and oxaliplatin with or without onartuzumab in HER2-negative, MET-positive gastroesophageal adenocarcinoma: the metgastric randomized clinical trial. JAMA Oncology 3(5), 620-627 (2017). 
20 Keytruda (pembrolizumab) prescribing information. www.accessdata.fda.gov/drugsatfda_docs/label/2016/125514s012lbl.pdf

21 FDA grants accelerated approval to pembrolizumab for advanced gastric cancer. www.fda.gov/Drugs/InformationOnDrugs/ApprovedDrugs/ucm577093.htm

22 Derks S, Liao X, Chiaravalli AM et al. Abundant PD-L1 expression in Epstein-Barr virus-infected gastric cancers. Oncotarget 7(22), 32925-32932 (2016).

23 Kwok G, Yau TC, Chiu JW, Tse E, Kwong YL. Pembrolizumab (Keytruda). Hum. Vaccin. Immunother. 12(11), 2777-2789 (2016).

24 Patnaik A, Kang SP, Rasco D et al. Phase I study of pembrolizumab (MK-3475; anti-PD-1 monoclonal antibody) in patients with advanced solid tumors. Clin. Cancer. Res. 21(19), 4286-4293 (2015).

- $\quad$ First-in-human Phase I study of pembrolizumab in advanced solid tumors.

25 Ahamadi M, Freshwater T, Prohn M et al. Model-based characterization of the pharmacokinetics of pembrolizumab: a humanized anti-PD-1 monoclonal antibody in advanced solid tumors. CPT Pharmacometrics Syst. Pharmacol. 6(1), 49-57 (2017).

26 Freshwater T, Kondic A, Ahamadi M et al. Evaluation of dosing strategy for pembrolizumab for oncology indications. J. Immunother Cancer 5, 43 (2017).

27 Hamid O, Robert C, Daud A et al. Safety and tumor responses with lambrolizumab (anti-PD-1) in melanoma. N. Engl. J. Med. 369(2), 134-144 (2013).

28 Robert C, Schachter J, Long GV et al. Pembrolizumab versus ipilimumab in advanced melanoma. N. Engl. J. Med. 372(26), 2521-2532 (2015).

29 Garon EB, Rizvi NA, Hui R et al. Pembrolizumab for the treatment of non-small-cell lung cancer. N. Engl. J. Med. 372(21), 2018-2028 (2015).

30 Reck M, Rodriguez-Abreu D, Robinson AG et al. Pembrolizumab versus chemotherapy for PD-L1-positive non-small-cell lung cancer. N. Engl. J. Med. 375(19), 1823-1833 (2016).

31 Muro K, Chung HC, Shankaran V et al. Pembrolizumab for patients with PD-L1-positive advanced gastric cancer (KEYNOTE-012): a multicentre, open-label, Phase Ib trial. Lancet Oncol. 17(6), 717-726 (2016).

-• First trial that investigated use of pembrolizumab in advanced PD-L1+ GEA.

32 Doi T, Piha-Paul SA, Jalal SI et al. Updated results for the advanced esophageal carcinoma cohort of the Phase Ib KEYNOTE-028 study of pembrolizumab (MK-3475). J. Clin. Oncol. 34 (4 Suppl.), 7 (2016).

33 The Cancer Genome Atlas Research Network. Integrated genomic characterization of oesophageal carcinoma. Nature 541(7636), 169-175 (2017).

34 Fuchs CS, Doi T, Jang RW-J et al. KEYNOTE-059 cohort 1: Efficacy and safety of pembrolizumab (pembro) monotherapy in patients with previously treated advanced gastric cancer. J. Clin. Oncol. 35(15 Suppl.), 4003-4003 (2017).

- Phase II trial of third-line pembrolizumab in advanced GEA.

35 Wainberg ZA, Jalal S, Muro K et al. LBA28_PRKEYNOTE-059 update: efficacy and safety of pembrolizumab alone or in combination with chemotherapy in patients with advanced gastric or gastroesophageal (G/GEJ) cancer. Ann. Oncol. 28(Suppl. 5), mdx440.020 (2017).

36 Catenacci DV, Wainberg Z, Fuchs CS et al. LBA-009KEYNOTE-059 cohort 3: safety and efficacy of pembrolizumab monotherapy for first-line treatment of patients (pts) with PD-L1-positive advanced gastric/gastroesophageal (G/GEJ) cancer. Ann. Oncol. 28(Suppl. 3), mdx302.008 (2017).

- Phase II trial of first line pembrolizumab in advanced PD-L1+ GEA.

37 Bang Y-J, Muro K, Fuchs CS et al. KEYNOTE-059 cohort 2: safety and efficacy of pembrolizumab (pembro) plus 5-fluorouracil (5-FU) and cisplatin for first-line (1L) treatment of advanced gastric cancer. J. Clin. Oncol. 35(Suppl. 15), 4012-4012 (2017).

- Phase II trial of first line combination therapy with pembrolizumab and chemotherapy in advanced GEA.

38 Chau I, Bendell JC, Calvo E et al. Interim safety and clinical activity in patients (pts) with advanced gastric or gastroesophageal junction (G/GEJ) adenocarcinoma from a multicohort Phase I study of ramucirumab (R) plus pembrolizumab (P). J. Clin. Oncol. 35(4 Suppl.), 102-102 (2017).

39 Tabernero J, Bang Y-J, Fuchs CS et al. KEYNOTE-062: Phase III study of pembrolizumab (MK-3475) alone or in combination with chemotherapy versus chemotherapy alone as first-line therapy for advanced gastric or gastroesophageal junction (GEJ) adenocarcinoma. J. Clin. Oncol. 34(4 Suppl.), TPS185-TPS185 (2016).

40 First-line esophageal carcinoma study with chemo vs. chemo plus pembrolizumab (MK-3475-590/KEYNOTE-590). https://clinicaltrials.gov/ct2/show/NCT03189719

41 Phase II trial of pembrolizumab with trastuzumab and chemotherapy in advanced HER2 positive esophagogastric (EG) cancer. https://clinicaltrials.gov/ct2/show/record/NCT02954536

42 Pembrolizumab, trastuzumab, HER2 positive gastric cancer. https://clinicaltrials.gov/ct2/show/record/NCT02901301 
43 Ohtsu A, Tabernero J, Bang Y-J et al. Pembrolizumab (MK-3475) versus paclitaxel as second-line therapy for advanced gastric or gastroesophageal junction (GEJ) adenocarcinoma: Phase III KEYNOTE-061 study. J. Clin. Oncol. 34(4 Suppl.), TPS183-TPS183 (2016).

44 Doi T, Bennouna J, Shen L et al. KEYNOTE-181: Phase 3, open-label study of second-line pembrolizumab vs single-agent chemotherapy in patients with advanced/metastatic esophageal adenocarcinoma. J. Clin. Oncol. 34(15 Suppl.), TPS4140-TPS4140 (2016).

45 Bang YJ, Giaccone G, Im SA et al. First-in-human Phase 1 study of margetuximab (MGAH22), an Fc-modified chimeric monoclonal antibody, in patients with HER2-positive advanced solid tumors. Ann. Oncol. 28(4), 855-861 (2017).

46 Catenacci DVT, Kim SS, Gold PJ et al. A Phase 1b/2, open label, dose-escalation study of margetuximab (M) in combination with pembrolizumab (P) in patients with relapsed/refractory advanced HER2+ gastroesophageal (GEJ) junction or gastric (G) cancer. J. Clin. Oncol. 35 (4 Suppl.), TPS219-TPS219 (2017).

47 Shah MA, Bennouna J, Shen L et al. Pembrolizumab (MK-3475) for previously treated metastatic adenocarcinoma or squamous cell carcinoma of the esophagus: Phase II KEYNOTE-180 study. J. Clin. Oncol. 34 (4 Suppl.), TPS189-TPS189 (2016).

48 Deng L, Liang H, Burnette B et al. Irradiation and anti-PD-L1 treatment synergistically promote antitumor immunity in mice. J. Clin. Invest. 124(2), 687-695 (2014).

49 Chao J, Chen Y-J, Frankel PH et al. Combining pembrolizumab and palliative radiotherapy in gastroesophageal cancer to enhance anti-tumor T-cell response and augment the abscopal effect. J. Clin. Oncol. 35 (4 Suppl.), TPS220-TPS220 (2017).

50 Phase $1 \mathrm{~b}$ open-label study of PEGPH20 with pembrolizumab. https://clinicaltrials.gov/ct2/show/record/NCT02563548

51 Safety and efficacy of CRS-207 with pembrolizumab in gastric, gastroesophageal junction or esophageal cancers. https://clinicaltrials.gov/ct2/show/NCT03122548

52 Rimm DL, Han G, Taube JM et al. A prospective, multi-institutional, pathologist-based assessment of 4 immunohistochemistry assays for PD-L1 expression in non-small cell lung cancer. JAMA Oncol. 3(8), 1051-1058 (2017).

53 Tran PN, Sarkissian S, Chao J, Klempner SJ. PD-1 and PD-L1 as emerging therapeutic targets in gastric cancer: current evidence. Gastrointestinal Cancer 7, 1-11 (2017).

54 List of cleared or approved companion diagnostic devices (in vitro and imaging tools). www.fda.gov/MedicalDevices/ProductsandMedicalProcedures/InVitroDiagnostics/ucm301431.htm

55 Cancer Genome Atlas Research Network. Comprehensive molecular characterization of gastric adenocarcinoma. Nature 513(7517), 202-209 (2014).

-• Molecular characteristics of primary untreated gastric adenocarcinomas as part of The Cancer Genome Atlas project.

56 Farris AB 3rd, Demicco EG, Le LP et al. Clinicopathologic and molecular profiles of microsatellite unstable Barrett Esophagus-associated adenocarcinoma. Am. J. Surg. Pathol. 35(5), 647-655 (2011).

57 Zhu L, Li Z, Wang Y, Zhang C, Liu Y, Qu X. Microsatellite instability and survival in gastric cancer: a systematic review and meta-analysis. Mol. Clin. Oncol. 3(3), 699-705 (2015).

58 Smyth EC, Wotherspoon A, Peckitt C et al. Mismatch repair deficiency, microsatellite instability, and survival: an exploratory analysis of the Medical Research Council Adjuvant Gastric Infusional Chemotherapy (MAGIC) Trial. JAMA Oncol. 3(9), 1197-1203 (2017).

59 Gryfe R, Kim H, Hsieh ET et al. Tumor microsatellite instability and clinical outcome in young patients with colorectal cancer. N. Engl. J. Med. 342(2), 69-77 (2000).

60 Dudley JC, Lin MT, Le DT, Eshleman JR. Microsatellite instability as a biomarker for PD-1 blockade. Clin. Cancer. Res. 22(4), $813-820$ (2016).

61 Le DT, Uram JN, Wang H et al. PD-1 blockade in tumors with mismatch-repair deficiency. N. Engl. J. Med. 372(26), 2509-2520 (2015).

62 Diaz LA, Marabelle A, Delord J-P et al. Pembrolizumab therapy for microsatellite instability high (MSI-H) colorectal cancer (CRC) and non-CRC. J. Clin. Oncol. 35(15 Suppl.), 3071-3071 (2017).

63 FDA approves first cancer treatment for any solid tumor with a specific genetic feature. www.fda.gov/newsevents/newsroom/pressannouncements/ucm560167.htm.

64 Camargo MC, Kim WH, Chiaravalli AM et al. Improved survival of gastric cancer with tumour Epstein-Barr virus positivity: an international pooled analysis. Gut 63(2), 236-243 (2014).

65 Kim SY, Park C, Kim HJ et al. Deregulation of immune response genes in patients with Epstein-Barr virus-associated gastric cancer and outcomes. Gastroenterology 148(1), 137.e139-147.e139 (2015).

66 Garcia-Diaz A, Shin DS, Moreno BH et al. Interferon Receptor Signaling Pathways Regulating PD-L1 and PD-L2 Expression. Cell Rep. 19(6), 1189-1201 (2017).

67 Ribas A, Robert C, Hodi FS et al. Association of response to programmed death receptor 1 (PD-1) blockade with pembrolizumab (MK-3475) with an interferon-inflammatory immune gene signature. J. Clin. Oncol. 33(15 Suppl.), 3001-3001 (2015). 
68 Carbone DP, Reck M, Paz-Ares L et al. First-line nivolumab in stage IV or recurrent non-small-cell lung cancer. N. Engl. J. Med. 376(25), 2415-2426 (2017).

69 Alexandrov LB, Nik-Zainal S, Siu HC, Leung SY, Stratton MR. A mutational signature in gastric cancer suggests therapeutic strategies. Nat. Commun. 6, 8683 (2015).

$70 \mathrm{Kim}$ JW, Nam KH, Ahn SH et al. Prognostic implications of immunosuppressive protein expression in tumors as well as immune cell infiltration within the tumor microenvironment in gastric cancer. Gastric Cancer 19(1), 42-52 (2016).

71 Kang BW, Seo AN, Yoon S et al. Prognostic value of tumor-infiltrating lymphocytes in Epstein-Barr virus-associated gastric cancer. Ann. Oncol. 27(3), 494-501 (2016).

72 Giampieri R, Maccaroni E, Mandolesi A et al. Mismatch repair deficiency may affect clinical outcome through immune response activation in metastatic gastric cancer patients receiving first-line chemotherapy. Gastric Cancer 20(1), 156-163 (2017).

73 Thompson ED, Zahurak M, Murphy A et al. Patterns of PD-L1 expression and CD8 T cell infiltration in gastric adenocarcinomas and associated immune stroma. Gut 66(5), 794-801 (2017).

74 Kang BW, Kim JG, Lee IH, Bae HI, Seo AN. Clinical significance of tumor-infiltrating lymphocytes for gastric cancer in the era of immunology. World. J. Gastrointest. Oncol. 9(7), 293-299 (2017).

75 Janjigian YY, Bendell JC, Calvo E et al. CheckMate-032: Phase I/II, open-label study of safety and activity of nivolumab (nivo) alone or with ipilimumab (ipi) in advanced and metastatic (A/M) gastric cancer (GC). J. Clin. Oncol. 34 (15 Suppl.), 4010-4010 (2016).

76 Kang Y-K, Satoh T, Ryu M-H et al. Nivolumab (ONO-4538/BMS-936558) as salvage treatment after second or later-line chemotherapy for advanced gastric or gastro-esophageal junction cancer (AGC): a double-blinded, randomized, Phase III trial. J. Clin. Oncol. 35(4 Suppl.), 2-2 (2017).

77 Boku N, Kang YK, Satoh T et al. 617OA Phase 3 Study of nivolumab (nivo) in previously treated advanced gastric or gastroesophageal junction (G/GEJ) cancer: updated results and subset analysis by PD-L1 expression (ATTRACTION-02). Ann. Oncol. 28(Suppl. 5), $\mathrm{mdx} 369.001-\mathrm{mdx} 369.001$ (2017).

78 Chung HC, Arkenau H-T, Wyrwicz L et al. Avelumab (MSB0010718C; anti-PD-L1) in patients with advanced gastric or gastroesophageal junction cancer from JAVELIN solid tumor Phase Ib trial: analysis of safety and clinical activity. J. Clin. Oncol. $34(15$ Suppl.), 4009-4009 (2016).

79 Segal NH, Antonia SJ, Brahmer JR et al. Preliminary data from a multi-arm expansion study of MEDI4736, an anti-PD-L1 antibody. J. Clin. Oncol. 32(15 Suppl.), 3002-3002 (2014).

80 Keytruda (pembrolizumab).

www.ema.europa.eu/docs/en_GB/document_library/EPAR_-_Product_Information/human/003820/WC500190990.pdf 